\title{
A Graph Method for Keyword-based Selection of the top-K Databases
}

\author{
Quang Hieu Vu ${ }^{1}$, Beng Chin Ooi ${ }^{1}$, Dimitris Papadias ${ }^{2}$, Anthony K. H. Tung ${ }^{1}$ \\ hieuvq@nus.edu.sg, ooibc@comp.nus.edu.sg, dimitris@cs.ust.hk, atung@comp.nus.edu.sg \\ ${ }^{1}$ National University of Singapore *, ${ }^{2}$ Hong Kong University of Science and Technology ${ }^{\dagger}$
}

\begin{abstract}
While database management systems offer a comprehensive solution to data storage, they require deep knowledge of the schema, as well as the data manipulation language, in order to perform effective retrieval. Since these requirements pose a problem to lay or occasional users, several methods incorporate keyword search (KS) into relational databases. However, most of the existing techniques focus on querying a single DBMS. On the other hand, the proliferation of distributed databases in several conventional and emerging applications necessitates the support for keyword-based data sharing and querying over multiple DMBSs. In order to avoid the high cost of searching in numerous, potentially irrelevant, databases in such systems, we propose $G-K S$, a novel method for selecting the top- $K$ candidates based on their potential to contain results for a given query. $G-K S$ summarizes each database by a keyword relationship graph, where nodes represent terms and edges describe relationships between them. Keyword relationship graphs are utilized for computing the similarity between each database and a KS query, so that, during query processing, only the most promising databases are searched. An extensive experimental evaluation demonstrates that $G$ - $K S$ outperforms the current state-of-the-art technique on all aspects, including precision, recall, efficiency, space overhead and flexibility of accommodating different semantics.
\end{abstract}

\section{INTRODUCTION}

Users of conventional DBMSs should have good knowledge of both the database schema and the data manipulation language (e.g., SQL) in order to effectively issue queries. Since the schema and the query language may be complex, database systems are often limited to advanced users. Several methods aim at decreasing this complexity by providing keyword-search (KS) functionality over a single database [2,

* Supported in part by ASTAR SERC Grant 0721010017 as part of S3 project [21].

${ }^{\dagger}$ Supported by grant HKUST 6184/06E from Hong Kong RGC.

Permission to make digital or hard copies of all or part of this work for personal or classroom use is granted without fee provided that copies are not made or distributed for profit or commercial advantage and that copies bear this notice and the full citation on the first page. To copy otherwise, to republish, to post on servers or to redistribute to lists, requires prior specific permission and/or a fee.

ACM SIGMOD '08 Vancouver, BC, Canada

Copyright 200X ACM X-XXXXX-XX-X/XX/XX ...\$5.00.

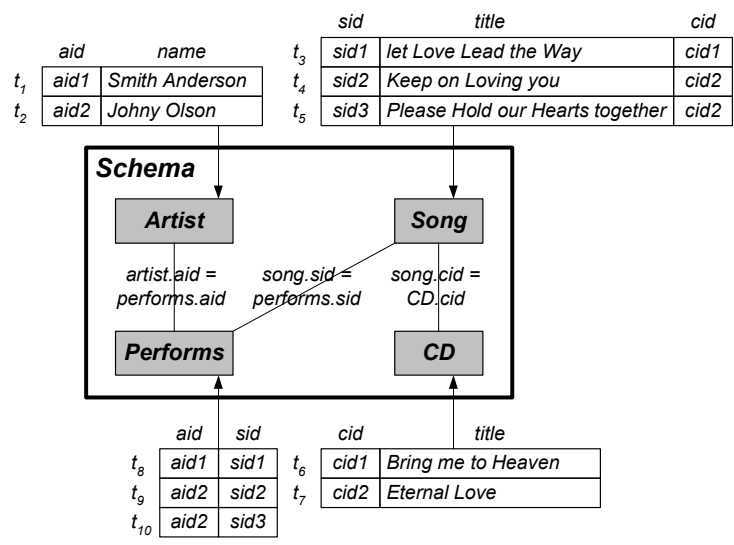

Figure 1: Running example of a music database

$1,13,12,14,16,11,18]$. In relational KS the basic unit of information is a tuple and each result is a set of tuples that (i) contain all (or most) keywords, and (ii) can be joined together in a meaningful way (usually on Primary Key Foreign Key relationships). This contrasts traditional information retrieval (IR), where each result is a single document (i.e., a basic unit of information).

We illustrate relational KS using the music database $D B_{1}$ of Figure 1, where edges between the tables correspond to join conditions. Given the KS query $q=\{$ Anderson, love $\}$, the system should return a result $t_{1} \bowtie t_{8} \bowtie t_{3}$, signifying that there is an artist called Anderson $\left(t_{1}\right)$ who performs $\left(t_{8}\right)$ a song $\left(t_{3}\right)$ containing love in its title. Note that although the term love also appears in $t_{4}$ and $t_{7}$, these tuples do not generate results as they cannot be connected to a record (i.e., $\left.t_{1}\right)$ containing Anderson. In the general case, there may exist numerous results, which can be ranked according to various criteria. A common scoring criterion used in all existing techniques is the distance ${ }^{1}$, i.e., the number of joins between two tuples containing the query keywords. In $D B_{1}$, the distance between $t_{1}$ and $t_{3}$ is 2 . Intuitively, the smaller the distance the more related the tuples, and therefore, the higher the importance of the corresponding result.

\footnotetext{
${ }^{1}$ Note that the distance is not constrained by the schema, but depends on the specific table instances. For example, in the schema of Figure 1 tuples could be connected by chains of more than 3 joins through intermediate records that belong to the same table. Most relational KS systems (e.g., $[13,26])$ use a distance threshold to eliminate long chains of joins, which usually lead to uninteresting results.
} 
Relational KS liberates the user from having to learn SQL and study the database schema (i.e., the tables, the attributes, and their possible connections). On the other hand, as keywords may appear in arbitrary columns (e.g., the term love may exist in the title of a song or an album, or even in the name of an artist), query processing is significantly more expensive than conventional DBMSs because all possible combinations of keyword co-occurrences have to be explored. The advantages (i.e., flexibility) and shortcomings (i.e., efficiency) of relational KS are amplified in distributed systems integrating multiple databases $D B_{1}$, ..,D $D B_{D}$. For instance, consider a group of universities that wish to share their library databases. Keyword based data sharing and search render the different schemas transparent, so that users can issue KS queries using a common interface without any knowledge of the underlying structure of $D B_{1}$, .., $D B_{D}$. However, processing each query in all the individual databases, and then combining the partial results, could be very costly and potentially unnecessary, especially if the final answers exist in a small subset of the databases.

In traditional IR, distributed systems alleviate the efficiency issue by pre-computing a term summary for each document repository. Given a query $q$, the system can select the most appropriate collections for processing $q$ based on the similarity between $q$ and the summaries. In other words, summaries act as documents in the corpus formed by all repositories and provide a fast mechanism for filtering out non-promising sources. However, IR methods are insufficient for relational KS as the mere presence of the query keywords in a summary is not indicative of the existence of results in the corresponding database; in addition, the tuples containing these keywords should be connected in a meaningful way. Assume for instance, a system integrating $D B_{1}$ of Figure 1 with another database, say $D B_{2}$, that includes the same tables and tuples as $D B_{1}$ except for $t_{8}$. Given $q=\{$ Anderson, love $\}$, both summaries of $D B_{1}$ and $D B_{2}$ contain similar statistical information (e.g., frequency, inverse term frequency) for the keywords Anderson and love, implying that they would be equally good candidates for search. However, the absence of $t_{8}$ in $D B_{2}$ eliminates the result $t_{1} \bowtie t_{8} \bowtie t_{3}$. In general, IR-based methods do not capture the inherent structure of a DBMS because they ignore connectivity and distance information among tuples containing terms.

In this paper, we aim at selecting the top- $K$ databases for processing a KS query, where $K$ is an input parameter. In accordance with the methodology of distributed IR techniques, we assume a system that pre-processes and maintains summaries of several DBMSs $D B_{1}, D B_{2}, . ., D B_{D}$. Given a KS query $q$, the system directs $q$ only to the $K<D$ databases most likely to contribute results, in order to minimize to total processing cost of $q$ without sacrificing precision and recall. The only existing technique focusing on the same problem is $M-K S$ [26], which is based on the concept of the keyword relationship matrix (KRM). Specifically, at a pre-processing phase, $M-K S$ builds a $K R M_{l}$ for every $D B_{l}$, which acts as its summary. For each term pair $\left(k_{i}, k_{j}\right)$ there is an entry $K R M_{l}\left(k_{i}, k_{j}\right)$ that records the frequencies of occurrences of the two terms at different distances. Given the 14 terms in $D B_{1}{ }^{2}, K R M_{1}$ would contain $14 \times 14$ entries. The

${ }^{2}$ Terms start with capital letters in Figure 1. Note that let, the, on, you, our, together, me, to are stop words, and hence they are not considered terms. entry $K R M_{1}($ Olson,love $)$ stores that the two terms can be connected once at distance 2 (i.e., $t_{2} \bowtie t_{9} \bowtie t_{4}$ ) and once at distance $3\left(t_{2} \bowtie t_{10} \bowtie t_{5} \bowtie t_{7}\right)$. The similarity between a query $q$ and $D B_{l}$ is computed using the $K R M_{l}$ entries of all possible keyword pairs in $q$; e.g., if $q=\left\{k_{1}, k_{2}, k_{3}\right\}$, the score of $D B_{l}$ is based on $K R M_{l}\left(k_{1}, k_{2}\right), K R M_{l}\left(k_{1}, k_{3}\right)$ and $K R M_{l}\left(k_{2}, k_{3}\right)$.

Despite its significant performance gains with respect to the naive solution of processing a KS query in all databases, $M-K S$ has several disadvantages. First, it uses only binary relationships between keyword terms to eliminate nonpromising databases. Consequently, it yields numerous false positives for queries where all pairs of keywords are related, but there is no join sequence linking the binary connections in a single result. Second, since $K R M$ records only the frequency of term co-occurrences (but no additional statistics), $M-K S$ is unsuitable for ranking based on IR measures (e.g., those in [16]). Finally, $M-K S$ does not include a mechanism for handling OR semantics. Therefore, it cannot retrieve (potentially interesting) answers even when a single keyword is missing.

To overcome these shortcomings, we propose $G-K S$, a graph based method for supporting keyword based data sharing and search over multiple databases. $G-K S$ summarizes each database $D B_{l}$ as a keyword relationship graph $K R G_{l}$ that captures the terms and their relationships with weighted nodes and edges. $K R G_{l}$ minimizes the chance of false positives by imposing more stringent conditions than simple binary relationships. Based on $K R G_{l}, G-K S$ can effectively estimate the importance of $D B_{l}$ with respect to a query under both the AND and the OR semantics. Our experiments show that, compared to $M-K S, G-K S$ (i) is more effective (improving recall/precision by as much as 50\%), (ii) is more efficient (reducing query processing cost by as much as $50 \%$ ), (iii) incurs less pre-processing time (faster by $30 \%$ ), and (iv) has less space overhead (smaller by $17 \%$ ). Our contributions are summarized as follows:

- We propose $G-K S$, a novel graph-based method for relational KS over multiple databases. We show how to construct, maintain and compress the KRGs in order to reduce the pre-processing and storage overhead.

- We present an IR-inspired method to compute the importance of nodes and edges, and an algorithm to estimate the potential of a join solution containing all query keywords.

- We confirm the efficiency and effectiveness of $G$ - $K S$ through extensive experiments with real datasets in a client-server architecture.

The rest of the paper is organized as follows. Section 2 , discusses related work. Section 3, presents the keyword relationship graph model. Section 4 describes the construction and maintenance of KRGs. Section 5 introduces the query processing algorithm and discusses the advantages of $G-K S$ over $M$-KS. Section 6 compares the two methods experimentally and Section 7 concludes the paper.

\section{RELATED WORK}

Section 2.1 overviews related work on KS over a single system. Section 2.2 surveys KS on multiple data repositories. 


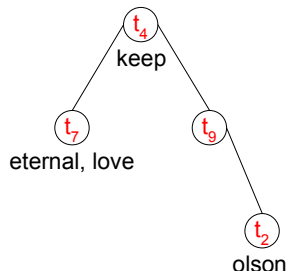

Figure 2: Connection tree for query \{Olson, keep, eternal, love\}

\subsection{Keyword search over a single system}

The majority of IR literature has focused on KS for unstructured documents stored at a single repository. Most methods are based on the Vector Space Model [24], which represents documents as term vectors [22] in the Cartesian Space. Each element in a vector captures a term and its weight, defined as $w=t f \cdot i d f$, where $t f$ is the term frequency in the document and $i d f$ is the inverse document frequency reflecting the general importance of the term in the entire corpus. Specifically, idf decreases the weight of terms that occur in numerous documents and, therefore, have low discriminating value (we refer to such terms as popular terms). Queries are also represented as vectors of keywords. The similarity of a query and a document is measured as the Cosine of the angle between their vector representations.

Several systems apply KS on a single relational DBMS. BANKS [2] creates a data graph, where each node represents a tuple, and edges connect tuples that can be joined (e.g., according to Primary Key - Foreign Key relationships). A KS query is processed by a graph traversal that searches for connection trees containing the query keywords. A connection tree is a Steiner tree in which every leaf node corresponds to a record containing at least one query keyword. Internal nodes represent tuples that connect the leaf records and may include no query keywords. Figure 2 shows a connection tree for query $q=\{$ Olson, keep, eternal, love $\}$ on the example database of Figure 1. Intuitively, the tree captures connectivity information for a result, in this case $t_{7} \bowtie t_{4} \bowtie t_{9} \bowtie$ $t_{2}$. BANKS employs a backward search strategy from leaf nodes containing the query keywords towards the root. On the other hand, [14, 11] apply bi-directional search, which improves efficiency. DBXplorer [1] and DISCOVER [13] use a higher level of representation - candidate networks created from the schema of the database by join operations. The systems use the candidate networks to generate operator trees for evaluating the query. Both DBXplorer and DISCOVER rank results based exclusively on the distance of the tuples containing the query keywords, whereas [12, 16, 18] utilize state-of-the-art IR measures to calculate scores.

Besides relational databases, KS has been applied in the context of XML databases $[10,5,15,25,17]$, where the search space is represented by a tree structure. Query results are the subtrees (or a part of the subtrees) of the XML document rooted at the lowest common ancestors (LCAs) [10], the semantic interconnection LCAs [5], the meaningful LCAs [15], or the smallest LCAs [25] of leaf nodes containing the query keywords. [17] presents a method to automatically select meaningful nodes in these subtrees. Recently, KS has also been extended to relational data streams [19], which necessitate the incorporation of temporal semantics and provision for frequent updates.

\subsection{Keyword search over distributed systems}

IR methods for KS over multiple repositories of documents generate summaries at a pre-processing phase. The summaries are utilized during query processing to filter out collections that cannot lead to good results. The existing techniques differ mainly on the way that they construct summaries. In the simplest form, GlOSS [9] and CVV [27] use only term frequencies to form summary information of each collection. CORI [3] adds a factor called inverse collection frequency, which reflects the importance of a term in all the repositories. Some other works $[20,8,4]$ also consider dependency relationships between terms (e.g., between occurrences of computer and programming). Although such relationships exist in natural languages [7], they are not applicable in relational KS. [23] proposes a method for relational $\mathrm{KS}$, where the tuples containing the query keywords may belong to different databases, assuming that records of distinct DBMSs can be joined. On the other hand, $M-K S$ [26] considers that the various databases are independent and the keywords must exist in the same DBMS to form a result. Since $M-K S$ focuses on the same setting as $G-K S$ and is our only competitor, in the sequel we provide a more detailed description.

$M-K S$ summarizes every $D B_{l}$ with a keyword relationship matrix $K R M_{l}$ capturing the binary relationships between terms at different distances. Specifically, each entry $K R M_{l}\left(k_{i}, k_{j}\right)$ stores a vector $d_{0} d_{1} \ldots$, where $d_{0}$ is the number of times that terms $k_{i}$ and $k_{j}$ occur in the same tuple, $d_{1}$ is the number of times that they occur in tuples with distance 1 and so on. In order to compute these vectors (for all term pairs), $M-K S$ scans $D B_{l}$, parses each tuple, removes stop words, stems the terms and inserts them into a table $T_{0}$ associating each term with the tuples that contain it. The $d_{0}$ values are obtained using $T_{0}$. Then, it joins all tables on foreign key relationships to generate a table $T_{1}$ with tuples at distance 1 ; based on $T_{1}$ and $T_{0}$, $M-K S$ derives $d_{1}$ for all term pairs. Similarly, $T_{2}$ is produced by self-joining $T_{1}$, and is used to obtain $d_{2}$. For $m>2, T_{m}$ is the result of $T_{m-1} \bowtie T_{1}$ after excluding tuples that exist in $T_{1}, . ., T_{m-1}$. The similarity between a query $q$ and $D B_{l}$ is based on the $K R M_{l}$ entries of all possible keyword pairs in $q$. Assuming that $q=\left\{k_{1}, k_{2}, k_{3}\right\}$, $M-K S$ retrieves $K R M_{l}\left(k_{1}, k_{2}\right), K R M_{l}\left(k_{1}, k_{3}\right), K R M_{l}\left(k_{2}, k_{3}\right)$ and assigns binary weights that reflect both the frequency of the co-occurrences and their distances (co-occurrences at smaller distance have a larger weight). The score of $D B_{l}$ is computed as a function (e.g., sum, product, minimum) of the binary weights.

\section{KEYWORD RELATIONSHIP GRAPHS}

$G-K S$ summarizes the terms and their relationships in each DBMS using a keyword relationship graph $(K R G)$. Figure 3 illustrates the $K R G$ for the example database of Figure 1. A node corresponds to a term and has a weight, which reflects its significance relative to other terms in the database. If two terms $k_{i}$ and $k_{j}$ exist in tuples $t_{x}$ and $t_{y}$ that can be connected through a sequence of joins, there is an edge between their corresponding nodes in the graph. The distance $d$ between $t_{x}$ and $t_{y}$ (in terms of join ${ }^{3}$ operations) is marked on the edge. When $k_{i}$ and $k_{j}$ can be

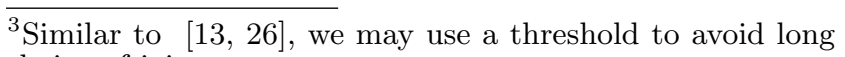
chains of joins. 


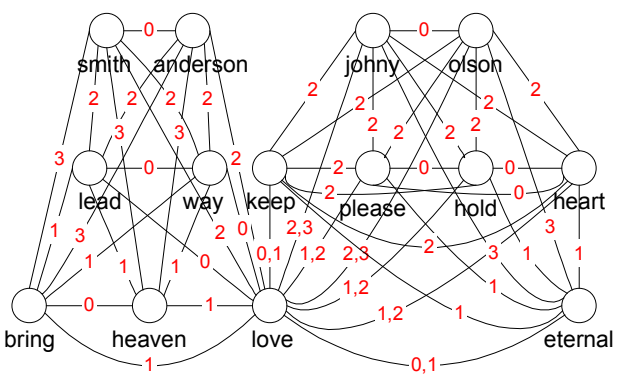

Figure 3: $K R G$ for the example database

connected through multiple paths of variable distances, each distinct value of $d$ is recorded. For instance, the edge between Olson and love has two values: 2 due to the result $t_{2} \bowtie t_{9} \bowtie t_{4}$, and 3 due to $t_{2} \bowtie t_{10} \bowtie t_{5} \bowtie t_{7}$. Every distance value in the graph is associated with a weight ${ }^{4}$ that measures the importance of the connection. As opposed to $M-K S$ that considers only frequency information, $G-K S$ utilizes IR-inspired measures to assign weights. Sections 3.1 and 3.2 discuss the computation of weights for nodes and edges, respectively. Section 3.3 presents a method for compressing the $K R G$. Table 1 summarizes the frequent symbols used throughout this paper for easy reference.

\subsection{Weight of a node}

Let $D B$ be a database and $k_{i}$ be a term appearing in a tuple $t$ of $D B$. We use $c_{i}(t)$ to denote the number of occurrences of $k_{i}$ in $t$. The size of tuple $t$ is $S(t)=\sum_{k} c_{k}(t) . N$ is the cardinality of tuples that include terms in $D B$, and $N_{i}$ is the number of tuples containing $k_{i}$.

DeFinition 1. Term frequency, $t f_{i}(t)$

The term frequency of $k_{i}$ in tuple $t$ is $f_{i}(t)=\frac{c_{i}(t)}{S(t)}$.

DeFINITION 2. Inverse tuple frequency, iuf $f_{i}$

The inverse tuple frequency of term $k_{i}$ is iuf $f_{i}=\ln \frac{N+1}{N_{i}}$.

Intuitively, $t f_{i}(t)$ and $i u f_{i}$ are analogous to term frequency and inverse document frequency in the Vector Space Model [24]. For instance, the sample database of Figure 1 contains $N=7$ tuples with terms $\left(t_{1}\right.$ to $\left.t_{7}\right)$. The term Anderson has a single appearance in $t_{1}$ (i.e., $N_{\text {anderson }}=1$ and $c_{\text {Anderson }}\left(t_{1}\right)=1$ ), which contains 2 terms in total (i.e., $S\left(t_{1}\right)$ $=2)$. Given the above, $t f_{\text {anderson }}\left(t_{1}\right)=\frac{1}{2}$ and $i u f_{\text {Anderson }}$ $=\ln \left(\frac{8}{1}\right)$.

Definition 3. Term weight, $w_{i}(t)$, Node weight, $w_{i}$ The weight of term $k_{i}$ in a tuple $t$ is $w_{i}(t)=t f_{i}(t) \cdot i u f_{i}$. The weight of the node representing $k_{i}$ is $w_{i}=\frac{\sum_{t=1}^{N_{i}} w_{i}(t)}{N_{i}}$.

Observe that $w_{i}(t)$ measures the importance of a term in a tuple, whereas $w_{i}$ captures the average score for a particular term among all tuples that contain it. Continuing the example, $w_{\text {Anderson }}\left(t_{1}\right)=t f_{\text {Anderson }}\left(t_{1}\right) \cdot i u f_{\text {Anderson }}=$ $\frac{1}{2} \cdot \ln \left(\frac{8}{1}\right)=1.040$. Since there is only one occurrence of the term in the database, the node representing Anderson in the $K R G$ of Figure 3 has a weight $w_{\text {Anderson }}=w_{\text {Anderson }}\left(t_{1}\right)$. Similarly, the term love appears in $N_{\text {love }}=3$ tuples $t_{3}, t_{4}, t_{7}$.

${ }^{4}$ For simplicity, we omit the node and edge weights in Figure 3 .
Table 1: Table of symbols

\begin{tabular}{|l|l|}
\hline Symbol & Definition \\
\hline \hline$t$ & tuple in a relational database \\
\hline$k$ & term in a relational database \\
\hline$n$ & node in a $K R G$ \\
\hline$d$ & distance relationship \\
\hline$t f$ & term frequency \\
\hline$i u f$ & inverse tuple frequency \\
\hline$p f$ & pairwise term frequency \\
\hline$i w f$ & inverse pairwise frequency \\
\hline$w$ & weight (of term, node, edge) \\
\hline
\end{tabular}

The sizes of these tuples are $S\left(t_{3}\right)=3, S\left(t_{4}\right)=2$ and $S\left(t_{7}\right)=2$, respectively. Consequently, the weight of the term in the $K R G$ is $w_{\text {love }}=$ average $\left(\frac{1}{3} \cdot \ln \left(\frac{8}{3}\right)+\frac{1}{2} \cdot \ln \left(\frac{8}{3}\right)+\frac{1}{2} \cdot \ln \left(\frac{8}{3}\right)\right)=$ 0.436 . The intuition behind $w_{\text {Anderson }}>w_{\text {love }}$ is that since Anderson exists in a single tuple, it has a larger discriminating value (and weight) than love, which occurs in 3 records (i.e., it is a popular term).

\subsection{Weight of an edge}

Let $t_{x}$ and $t_{y}$ be two tuples containing terms $k_{i}$ and $k_{j}$, respectively. These tuples may be connected by several paths of equal distances that pass through different intermediate records: $n c\left(t_{x}, t_{y}, d\right)$ is the number of unique connections between $t_{x}$ and $t_{y}$ at distance $d$, and $c_{i j}\left(t_{x}, t_{y}, d\right)=$ $n c\left(t_{x}, t_{y}, d\right) \cdot c_{i}\left(t_{x}\right) \cdot c_{j}\left(t_{y}\right)$ is the number of connections between $k_{i}$ and $k_{j}$ in $t_{x}$ and $t_{y}$. The values of $c_{i j}\left(t_{x}, t_{y}, d\right)$ and $n c\left(t_{x}, t_{y}, d\right)$ differ only if there are multiple occurrences of $k_{i}\left(k_{j}\right)$ in $t_{x}\left(t_{y}\right) . S_{i j}\left(t_{x}, t_{y}, d\right)=n c\left(t_{x}, t_{y}, d\right) \cdot S\left(t_{x}\right) \cdot S\left(t_{y}\right)$ is the number of connections between all term pairs in $t_{x}$ and $t_{y}$ at distance $d$.

Definition 4. Pairwise frequency, $p f_{i j}\left(t_{x}, t_{y}, d\right)$

The pairwise frequency of two terms $k_{i}$ and $k_{j}$ in tuples $t_{x}$ and $t_{y}$ at distance $d$ is $p f_{i j}\left(t_{x}, t_{y}, d\right)=\frac{c_{i j}\left(t_{x}, t_{y}, d\right)}{S_{i j}\left(t_{x}, t_{y}, d\right)}=$ $\frac{c_{i}\left(t_{x}\right) \cdot c_{j}\left(t_{y}\right)}{S\left(t_{x}\right) \cdot S\left(t_{y}\right)}=t f_{i}\left(t_{x}\right) \cdot t f_{j}\left(t_{y}\right)$.

Consider the terms Olson appearing in $t_{2}$ and love appearing in $t_{4}$. Recall that the two terms can be combined by two join operations connecting: $t_{2} \bowtie t_{9} \bowtie t_{4}$. Given that $t f_{\text {Olson }}\left(t_{2}\right)=t f_{\text {love }}\left(t_{4}\right)=0.5$, we obtain $p f_{\text {Olson }, \text { love }}\left(t_{2}, t_{4}, 2\right)=$ 0.25 . Note that $n c$ is canceled out in the computation of $p f_{i j}\left(t_{x}, t_{y}, d\right)$, and therefore, its existence is not necessary for the definition of pairwise frequency. We chose to include $n c$ for consistency with the previous section. Specifically, the fraction $\frac{c_{i j}\left(t_{x}, t_{y}, d\right)}{S_{i j}\left(t_{x}, t_{y}, d\right)}$ corresponds to $\frac{c_{i}(t)}{S(t)}$ used in the definition of the term frequency. Next, let $N_{i j}(d)$ be the total number of cases where two tuples containing $k_{i}$ and $k_{j}$ can be connected at distance $d$. Similarly, $N(d)$ is the total number of cases where two tuples containing any terms can be connected at distance $d$.

DEFINITION 5. Inverse pairwise frequency, $i w f_{i j}(d)$ The inverse pairwise frequency of two terms $k_{i}$ and $k_{j}$ at distance $d$ is iw $f_{i j}(d)=\ln \frac{N(d)+1}{N_{i j}(d)}$

Definition 6. Pairwise weight, $w_{i j}\left(t_{x}, t_{y}, d\right)$, Edge weight, $w_{i j}(d)$

The pairwise weight of two terms $k_{i}$ and $k_{j}$ in tuples $t_{x}$ and $t_{y}$ at distance $d$ is $w_{i j}\left(t_{x}, t_{y}, d\right)=p f_{i j}\left(t_{x}, t_{y}, d\right) \cdot i w f_{i j}(d)$. The weight of the edge connecting $k_{i}$ and $k_{j}$ at distance $d$ is $w_{i j}(d)=\frac{\sum_{1}^{N_{i j}} w_{i j}\left(t_{x}, t_{y}, d\right)}{N_{i j}(d)}$. 


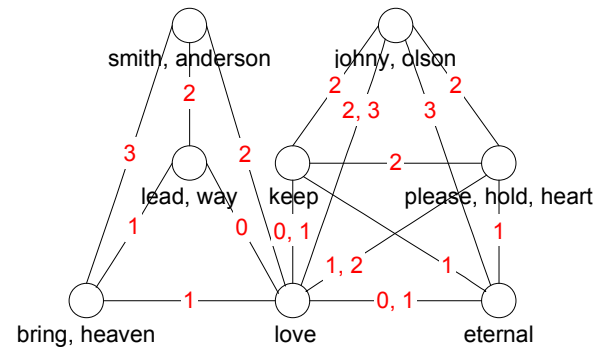

Figure 4: Compressed $K R G$

Intuitively, $w_{i j}(d)$ is the average weight among all pairs of tuples in the database that are connected at distance $d$ and include $k_{i}$ and $k_{j}$, respectively. Continuing our example, there is a single way to relate Olson and love at $d=2$; thus, $N_{\text {olson,love }}(2)=1$. The total number of cases where tuples (containing terms) are connected by 2 join operations is $N(2)=4\left(t_{1} \bowtie t_{8} \bowtie t_{3}, t_{2} \bowtie t_{9} \bowtie t_{4}, t_{2} \bowtie t_{10} \bowtie t_{5}\right.$, and $\left.t_{4} \bowtie t_{7} \bowtie t_{5}\right)$. Given the above: iwfolson,love $(2)=\ln \frac{5}{1}$. Consequently, $w_{\text {Olson,love }}\left(t_{2}, t_{4}, 2\right)=0.25 \cdot \ln \frac{5}{1}=0.402$. Since there is no other result involving these terms at $d=2$, the corresponding weight for edge of the $K R G$ in Figure 3 is $w_{\text {Olson,love }}(2)=w_{\text {Olson,love }}\left(t_{2}, t_{4}, 2\right)$.

\subsection{Graph compression}

Typically, a large percentage of the terms in a DBMS (around two thirds in our experiments) appear only once. If such terms occur in the same tuple: (i) they have the same weight; (ii) they have the same set of connections to other nodes; and (iii) these connections are of equal weight. Based on these observations we propose the following method for $K R G$ compression. During pre-processing, $G-K S$ identifies, for each tuple, the terms that appear only once in the database and inserts them in a compound node. Thus, the $K R G$ has two types of nodes: single nodes containing one term and compound nodes consisting of multiple terms. The weight of a compound node, or an edge involving a compound node, is calculated using any of the included terms. This simple method decreases significantly the size of the graph without incurring any information loss. Figure 4 shows the compressed graph for the $K R G$ of Figure 3. For instance, the terms Johny and Olson, which appear in $D B_{1}$ a single time in $t_{2}$, generate a compound node. Similarly, all three terms please, hold, heart of $t_{5}$ are unique and map to another compound node. In total, the reduced graph contains 8 nodes and 16 edges, compared to 14 nodes and 49 edges in the original $K R G$.

\section{GRAPH CONSTRUCTION AND MAIN- TENANCE}

Section 4.1 describes the initial construction of the keyword relationship graph. Section 4.2 discusses its maintenance in the presence of database updates.

\subsection{Graph construction}

The $K R G$ of a database is constructed in three steps. Initially, $G-K S$ extracts terms from the data tuples to create nodes for the $K R G$. In this step, the system creates a compound nodes for terms that occur only once in the database and in the same tuple. The remaining terms generate single nodes. At the end of this phase, weights are assigned based on the definitions of the previous section.

In the second step, the system constructs relationships between tuples in the database at different distances $d \geq 1$. We denote with $R_{T}(d)$ the set of tuple pairs $\left(t_{x}, t_{y}\right)$ that can be connected at distance $d$. $G$-KS inserts a pair $\left(t_{x}, t_{y}\right)$ in $R_{T}(1)$ if they have a Primary Key - Foreign key (PKFK) relationship. For $d>1,\left(t_{x}, t_{y}\right)$ is added to $R_{T}(d)$, if there exists a tuple $t_{z}$ such that (i) $t_{z}$ has a relationship with either $t_{x}$ or $t_{y}$ in $R_{T}(d-1)$ and (ii) $t_{z}$ can be joined with the other tuple of the pair. For instance, $\left(t_{x}, t_{y}\right) \in R_{T}(d)$, if $\left(t_{x}, t_{z}\right) \in R_{T}(d-1)$ and there is a PK-FK relationship between $t_{z}$ and $t_{y}$.

Finally, based on the relationships of tuples at different distances, $G-K S$ creates edges between nodes at the $K R G$. For each pair of nodes $\left(n_{i}, n_{j}\right)$, where $n_{i}$ represents a term in $t_{x}, n_{j}$ represents a term in $t_{y}$, and $\left(t_{x}, t_{y}\right) \in R_{T}(d)$, there is an edge between $n_{i}$ and $n_{j}$ at distance $d$. Note that if the nodes correspond to terms in the same tuple, the distance is 0 .

\subsection{Graph maintenance}

When new tuples are inserted or old tuples are deleted, the $K R G$ should be modified accordingly to reflect the current instance of the database. Since an update can be considered as a deletion followed by an insertion, we only discuss the maintenance of $K R G$ in case of insertions and deletions. When a tuple $t$ is inserted into the database, $G-K S$ extracts all the included terms. New terms lead to the creation of nodes in the $K R G$, while existing terms lead to recalculation of the weights of the corresponding nodes. Then, the system computes the distance relationship for all pairs $\left(t, t_{x}\right)$ using PK-FK relationships. Additionally, a new entry for each pair $\left(t_{x}, t_{y}\right)$ is created in $R_{T}\left(d_{1}+d_{2}\right)$, if there exist $\left(t, t_{x}\right) \in R_{T}\left(d_{1}\right)$ and $\left(t, t_{y}\right) \in R_{T}\left(d_{2}\right)$.

Similar to the process of graph construction, based on the updated relationships of tuples at different distances, $G-K S$ creates new relationships for the corresponding nodes. If these nodes were already connected at that distance, the corresponding weight is re-computed. Otherwise, a new (distance, weight) pair is added to the edge. If this is the first connection between the nodes, this is equivalent to adding an edge to the KRG. Finally, the system creates new relationships and updates the weight of the existing relationships at distance 0 for nodes representing terms in the inserted tuple.

When an existing record $t$ is deleted, $G-K S$ first extracts all terms in $t$ and identifies the set $I$ of nodes containing these terms. Let $I(d)$ be the set of nodes that have relationships with nodes in $I$ at distance $d$. For each value of $d$, $G-K S$ retrieves $I(d)$ and deletes relationships between nodes $n_{i}$ and $n_{j}$ at distance $d$ satisfying one of the following conditions (i) $n_{i}, n_{j} \in I \wedge d=0$ or (ii) $n_{i} \in I \wedge n_{j} \in I(d)$ or (iii) $n_{i} \in I\left(d_{1}\right) \wedge n_{j} \in I\left(d_{2}\right) \wedge d=d_{1}+d_{2}$. For the third case, note that although the deletion of $t$ may not affect $\left(n_{i}, n_{j}\right)$ (if the two tuples have multiple connections at $d$ ), this cannot be known since the $K R G$ does not maintain information about the specific paths. However, if $n_{i}$ and $n_{j}$ can be connected through a path not containing $t$, their relationship is recreated in the next step.

Next, all nodes that represent exclusively terms of $t$ are removed from the $K R G$ (i.e., the corresponding terms do not appear anywhere else in the databases) and the weights of 
the remaining nodes in $I$ are updated. Finally, let $T_{I}$ be the set of tuples containing the terms represented by nodes in $I$. Similarly, $T_{I}(d)$ is the set of tuples containing the terms corresponding to nodes in $I(d)$. $G-K S$ computes new distance relationships between all tuple pairs $\left(t_{x}, t_{y}\right)$ such that (i) $t_{x} \in T_{I}$ and $t_{y} \in T_{I}(d)$, or (ii) $t_{x} \in T_{I}\left(d_{1}\right)$ and $t_{y} \in T_{I}\left(d_{2}\right)$ and $d=\left(d_{1}+d_{2}\right)$. Then, it updates edge weights based on the new tuple relationships. Finally, $G-K S$ recreates relationships at distance 0 for nodes in $I$ representing terms in the same tuple.

In order to avoid the cost of frequent revisions, the $K R G$ may be updated in a batch mode periodically (e.g., at the end of each working day) or after the database modifications accumulate to a certain level. $G-K S$ processes batch insertions using the same method as for a single record, except that the extracted terms exist in any of the new tuples. For batch deletions, the system applies the methodology of single deletion considering all terms of the deleted tuples.

\section{QUERY PROCESSING}

Given a KS query $q$ and a set of $K R G s$, each created for a database, the goal of query processing is to find the top- $K$ databases for directing the query. Section 5.1 introduces the concepts of join keyword trees and candidate graphs used to evaluate the potential of a database. Section 5.2 proposes an algorithm for finding candidate graphs. Section 5.3 describes the ranking mechanism, and Section 5.4 discusses the advantages of $G$ - $K S$ over $M-K S$.

\subsection{Join keyword trees and candidate graphs}

Let $K R G$ be the keyword relationship graph of a database $D B$, and $S G$ be a sub graph of $K R G$. We evaluate the potential of $D B$ with respect to query $q$ based on the structure of the $K R G$. Towards this direction we define the concept of the join keyword tree $(J K T)$. To avoid confusion, we refer to a $J K T$ node as a vertex.

Definition 7. Join keyword tree, JKT $(S G)$

Given SG, JKT $(S G)$ is a tree satisfying the following properties:

1. Each tree vertex $t_{i}$ maps to a non-empty set of nodes of SG, and the tree vertices should collectively contain all nodes in $\mathrm{SG}$.

2. Edges connecting two vertices are associated with a single distance $d$.

3. If two $\mathrm{SG}$ nodes $\left(n_{i}, n_{i}^{\prime}\right)$ map to the same tree vertex $t n_{i}$, they must co-exist in some tuple of $\mathrm{DB}$. If there is an edge between two vertices $\left(t n_{i}, t n_{j}\right)$ with distance $d$ in JKT, then all pairs of corresponding nodes $n_{i} \in t n_{i}$ and $n_{j} \in t n_{j}$ must be related at $d$ in $\mathrm{SG}$. If two vertices $\left(t n_{i}, t n_{j}\right)$ are not directly connected in $\mathrm{JKT}$, then for each pair of nodes $n_{i} \in t n_{i}$ and $n_{j} \in t n_{j}$, there must be a relationship in SG at distance equal to that of the path connecting $t n_{i}$ and $t n_{j}$.

Figure 5 shows a $J K T$ of an $S G$ containing four nodes $n_{\text {johny,olson }}, n_{\text {keep }}, n_{\text {eternal }}$ and $n_{\text {love }}$ of the $K R G$ in Figure 4. This tree is a $J K T$ because (1) each vertex covers some node(s) existing in the $S G$ and all nodes appear in the tree vertices; (2) each edge has exactly one distance; (3) Eternal and love are in the same vertex tneternal,love as

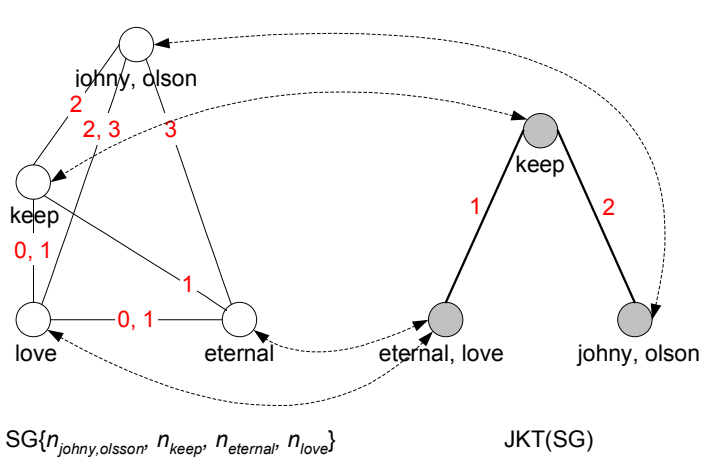

Figure 5: Mapping from $S G$ to $J K T(S G)$

they are related at distance 0 in the $S G$ (they co-exist in $\left.t_{7}\right)$. Vertices $t n_{\text {eternal,love }}$ and $t n_{\text {keep }}$ are directly connected by an edge with $d=1$ and there is a relationship at distance 1 for pairs $\left(n_{\text {eternal }}, n_{\text {keep }}\right)$ and $\left(n_{\text {love }}, n_{\text {keep }}\right)$ in the $S G$. Similarly, vertices $t n_{\text {johny,olson }}$ and $t n_{\text {keep }}$ are directly connected by an edge with $d=2$ and there is a relationship at distance 2 for the pair $\left(n_{\text {johny,olson }}, n_{\text {keep }}\right)$ in the $S G$. Finally, the length of the path between $t n_{\text {eternal,love }}$ and $t n_{\text {johny,olson }}$ is 3 , and there is a relationship at distance 3 for both $\left(n_{\text {eternal }}, n_{\text {johny,olson }}\right)$ and $\left(n_{\text {love }}, n_{\text {johny,olson }}\right)$ in the $S G$.

Let $q$ be a query that contains exactly the keywords represented by nodes in $S G \subseteq K R G$ and assume that the database corresponding to the $K R G$ has query results. A $J K T(S G)$ serves as a model for the construction of a connection tree of a result. In particular, a connection tree containing the query keywords can be built from $J K T$ by adding intermediate nodes between $J K T$ vertices connected through edges with distance greater than 1 . For example, given $q=\{$ Olson, $k e e p$, eternal, love $\}$, if we insert an intermediate node between $t n_{\text {keep }}$ and $t n_{\text {johny,olson }}$ in the $J K T$ in Figure 5 , we derive a graph that has the same structure with the connection tree of Figure 2. This indicates that if $K R G$ has an $S G$ containing the query keywords, and there exists a $J K T(S G)$, it is likely that the database contains a query result. Following this fact, we give a definition of the Candidate Graph $(C G)$ as follows.

Definition 8. Candidate graph, $C G(K R G, q)$

Given a query $q$ and a $\mathrm{KRG}, C G(K R G, q)$ is an $\mathrm{SG}$ of KRG satisfying the following properties

1. $S G$ includes all nodes of KRG containing the query keywords, and only these nodes.

2. $S G$ is complete (i.e., there is an edge between each pair of nodes).

3. There exists at least one $J K T(S G)$.

The $K R G$ of Figure 4 has a candidate graph with respect to the query $q=\{$ Olson, keep, eternal, love $\}$ because it includes a complete $S G$ containing the four keywords and there is a $J K T$ for this $S G$ as shown in Figure 5. Next we discuss the relationship between the existence of candidate graphs in $K R G$ and results in the corresponding database.

THEOREM 5.1. If a database contains a result with all keywords of query $q$, then the corresponding KRG must have a candidate graph $C G(K R G, q)$. 
Proof: Assume a database containing a result $t_{1} \bowtie t_{2} \bowtie$ ... The existence of the result implies that the corresponding $K R G$ has an $S G$, that contains a node for each query term. Since all keywords appear in $t_{1} \bowtie t_{2} \bowtie$.., then all possible keyword pairs must be connected in $S G$. Therefore, $S G$ satisfies the first two properties of a candidate graph. Next we show how to generate a $J K T$ of $S G$.

Based on the join sequence $t_{1} \bowtie t_{2} \bowtie$.. we create a $J K T$ using the following procedure. (i) Select a random tuple as a root. (ii) Pick another record, which can be joined with the first one, and insert it as a child of the root. (iii) While there are still unused tuples, pick a record $t_{i}$ (among the remaining ones) that can join with an inserted tuple $t_{j}$ and add $t_{i}$ as a child of $t_{j}$. (iv) Set the distance of each link in the tree to 1. (iv) Replace each tuple with the set of query keywords inside. (v) For every record $t_{k}$ that does not contain any keyword, remove $t_{k}$ from the tree, connect its parent and its children, and increase the distance of these links by 1 . The final output is a $J K T(S G)$.

Note that a join sequence may create multiple JKTs depending on the order of insertions in the tree. These JKTs are equivalent in the sense that they correspond to the same result. Theorem 5.1 can be used to filter out databases that are guaranteed not to include results (i.e. databases not having a candidate graph). Although the existence of $C G(K R G, q)$ is a necessary condition in order for $K R G$ to contain results, as shown in the following theorem, it is not sufficient. Furthermore, the proof of Theorem 5.2 provides important insight about the structure of false positives in $G-K S$, i.e., candidate graphs that fail to produce actual results.

THEOREM 5.2. The existence of a candidate graph $C G(K R G, q)$ in KRG does not guarantee that the corresponding database has results for $q$.

Proof: Let $n_{x}$ be a node of $C G(K R G, q)$ representing a query keyword $k_{x}$, which is mapped to a vertex $t n_{x}$ of a $J K T(C G) ; n_{y}$ is a $K R G$ node for term $k_{y}$ that does not belong to $C G$ (i.e., $k_{y}$ is not a query keyword). Assume that for each node $n_{i} \neq n_{x}, n_{i} \in C G$, if $n_{i}$ is mapped to the same tree vertex $t n_{x}$ as $n_{x}$, there exists a relationship between $n_{i}$ and $n_{y}$ at distance 0 . If $n_{i}$ is mapped to a tree vertex $t n_{i} \neq t n_{x}$, there exists a relationship between $n_{i}$ and $n_{y}$ at distance equal to the distance between $t n_{i}$ and $t n_{x}$ in the tree structure. In other words, if we replace $n_{x}$ with $n_{y}$ we obtain $J K T\left(S G^{\prime}\right)$, where $S G^{\prime}=\left(C G \backslash n_{x}\right) \cup n_{y}$. We further assume that: (1) $n_{x}$ and $n_{y}$ appear at different tuples $t_{x}$ and $t_{y}$ that are not connected; and (2) there are two nodes $n_{i}, n_{j}$ in $C G(K R G, q)$ related by a join through tuple $t_{y}$. Consequently, the actual join sequence forming the $J K T$ may be due to $S G^{\prime}$. In this case, there may not exist any result in the database containing all the keywords even though the $K R G$ has a candidate graph.

Figure 6(a) shows an updated instance of the music database of Figure 1, where there are new tuples $t_{11}$ to $t_{14}$. These tuples indicate that artist Paulo Anderson $\left(t_{11}\right)$ performs $\left(t_{14}\right)$ a song Wish $\left(t_{12}\right)$ in a CD entitled Something in my heart $\left(t_{13}\right)$. Now, let us consider a query $q=\{$ Anderson, love, heart $\}$. The $K R G$ includes a candidate graph because the $S G$ containing the query keywords is complete and has a $J K T(S G)$ as shown in Figure 6(b). However, although $C G(K R G, q)$ exists, the database does not have a result for this query. Observe that any join sequence originating

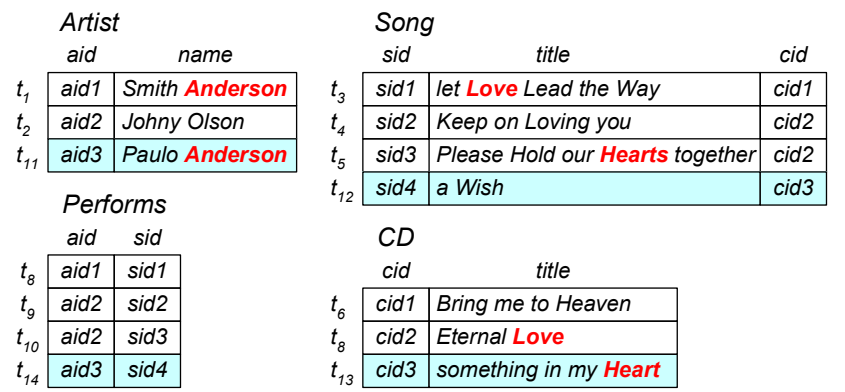

(a) The updated music database

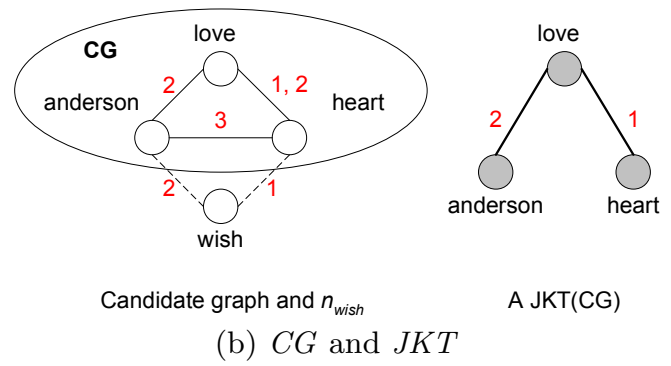

Figure 6: Candidate graph without result

from Paulo Anderson $\left(t_{11}\right)$ does not contain Love, whereas any sequence from Smith Anderson $\left(t_{1}\right)$ does not include heart. We demonstrate the reason for the failure by setting $k_{x}=$ love and $k_{y}=$ wish in the above proof. The vertices $t n_{\text {Anderson }}, t n_{\text {heart }}$ corresponding to Anderson and heart are related with $t n_{\text {love }}$ at distances 2 and 1 in $J K T$, respectively.

In $K R G, n_{\text {anderson }}, n_{\text {heart }}$ are connected with $n_{w i s h}$ at the same distance. Furthermore, wish and love exist in different tuples that are not connected at any distance. This implies that there is not way to distinguish if $n_{\text {anderson }}$ and $n_{\text {heart }}$ are connected through $n_{\text {love }}$, or through $n_{\text {wish }}$ (as is the case here) because $K R G$ (and consequently $J K T(S G)$ ) does not represent the paths between nodes, but only their distances. We refer to KRGs that contain candidate graphs, but not query results, as false positives.

Even though a candidate graph does not provide certainty, it does generally indicate a high probability of the database having results. Let us consider a query with $\kappa$ keywords and assume that each keyword is represented by a node in $K R G$. There are totally $C_{\kappa}^{2}$ relationships between pairs of nodes in the $S G$ containing the query keywords. To determine if the $S G$ is a candidate graph, we need to find a $J K T(S G)$. The third condition of the JKT definition goes beyond the binary relationships in $K R G$ and tests collectively all relationships as the whole. This is due to the fact that a test on a pair of vertices in the $J K T$ involves both (i) checking their direct relationship, and (ii) relationships between vertices in the path connecting them. Therefore, for a false positive to occur, the node $n_{y}$ of Theorem 5.2 has to satisfy $\kappa-1$ relationships to other nodes (to form a complete graph) and also $C_{\kappa}^{2}-(\kappa-1)$ hidden relationships between the remaining nodes. In most cases this probability is low and decreases as the number of query keywords increases. On the other hand, recall that $M-K S$ simply involves binary relationships and the chance of false positives increases with the query size. 


\subsection{Search for candidate graphs}

Given a query $q, G-K S$ retrieves the subgraph $S G$ of $K R G$ that contains all the query keywords and checks whether it is complete. If either keyword coverage, or completeness is violated, then $S G$ cannot be a candidate graph, implying that the corresponding database cannot lead to any result. This is similar to the filtering mechanism of $M-K S$. However, whereas $M-K S$ would consider successful all $S G s$ that feature keyword coverage and completeness, $G$ - $K S$ provides an additional level of filtering by verifying whether $S G$ is a candidate graph.

Algorithm 1, hereafter referred to as ValidateSG, determines whether a valid join keyword tree can be induced in $S G$. Since we only need a single $J K T$, we employ a depthfirst traversal ${ }^{5}$. Specifically, Validate $S G$ initializes a list $L$ containing all nodes in $S G$, randomly picks a node in $L$ as the root of a tree $T$, and pushes a pair of $(T, L)$ to a stack $S$ (lines 1-6). Then, it iteratively pops out $(T, L)$ from $S$ until either $S$ or $L$ is empty (lines 7-23). If $S$ is empty, no solution $J K T$ exists, and hence $S G$ is not a candidate graph. On the other hand, if $L$ is empty, all nodes have been assigned to a $J K T T$, and $S G$ is a candidate graph. In each of the repeated steps, Validate $S G$ selects a node $n_{x}$ among the remaining in $L$ and considers all positions for $n_{x}$ at the existing tree $T$. In particular, if there are $S_{n}$ vertices in $T$, there are $\left(3 \cdot S_{n}-1\right)$ possible locations for $n_{x}$ : (i) $S_{n}$ cases for inserting $n_{x}$ in the same vertex as an existing node $n_{i}$ (lines 14-16), (ii) $S_{n}$ cases of assigning $n_{x}$ as a child of a existing vertex (lines 17-19) and (iii) $S_{n}-1$ cases of inserting $n_{x}$ between two parent-child vertices in the tree (lines 21-23). If after the addition of $n_{x}$, the tree can lead to a $J K T$ i.e. vertices in the tree satisfy the third condition of a $J K T, T$ and $L$ are pushed to the stack $S$.

For simplicity, the pseudo-code presents only the basic functionality of ValidateSG. In addition, we employ two strategies to speed up the process of finding a JKT. First, instead of selecting nodes randomly, we sort them by their total number of different distances with respect to other nodes in $S G$. Then, at each step, we select the node with the minimum number of distances. This strategy reduces the possible valid positions for a new node (to be inserted in the tree) and minimizes the number of potential JKTs in the stack. Consequently, if the graph has no $J K T$, the process can stop after exploring a small number of potential trees. Furthermore, the first nodes in the list form a stable solution; therefore, when backtracking is necessary, the number of backtracking steps is relatively low. Second, instead of trying all possible positions when inserting a node into a tree, we only attempt to add $n_{x}$ around the existing node $n_{y}$ with the smallest distance to $n_{x}$. Recall that for the new tree to satisfy the requirements of the $J K T$ definition, the distance between $n_{x}$ and $n_{y}$ has to exceed the distance between $n_{x}$ and every intermediate node $n_{z}$ in the path connecting $n_{x}$ and $n_{y}$. This requirement cannot be satisfied if $n_{x}$ is attached to $n_{z}$.

As an example of the optimizations, consider searching for a $J K T$ in the graph of Figure 5 . Each of $n_{\text {keep }}, n_{\text {eternal }}$, $n_{\text {johny,olson }}$ has 4 possible distances to other nodes, while $n_{\text {love }}$ has 6 . We insert the nodes in a priority list $L_{P}=$

\footnotetext{
${ }^{5}$ There may exist numerous JKTs because two nodes can be connected at different distances, each leading to a different $J K T$.
}

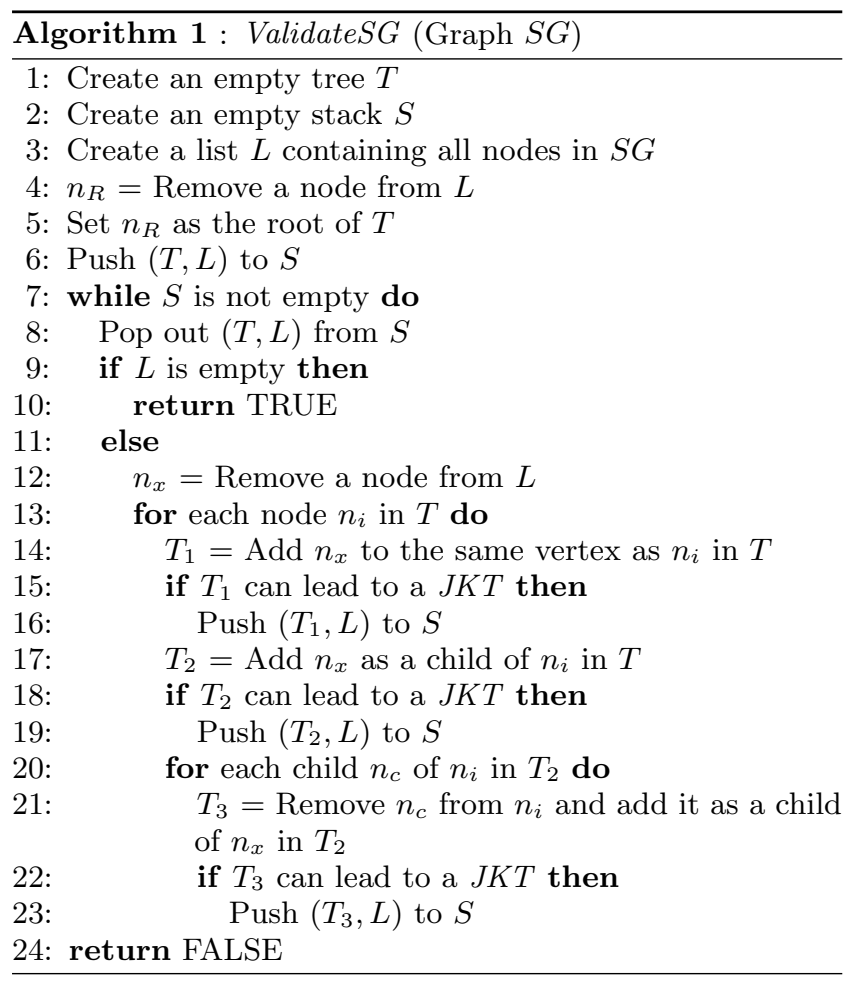

$\left(n_{\text {keep }}, n_{\text {eternal }}, n_{\text {johny,olson }}, n_{\text {love }}\right)$ (the first three nodes are ordered at random). Given $L_{P}$, the root of the tree is $n_{\text {keep }}$. Next, $n_{\text {eternal }}$ is inserted as a child of $n_{\text {keep }}$. Note that $n_{\text {eternal }}$ cannot be added to the same vertex as $n_{k e e p}$ because they are not related at distance 0 (therefore the resulting tree could not lead to JKT). During the insertion of $n_{\text {johny,olson }}$, the second optimization considers the positions around $n_{\text {keep }}$ (i.e., the node with the minimum distance to $\left.n_{\text {johny,olson }}\right)$. Observe that if we add $n_{\text {johny,olson }}$ as a child of $n_{\text {eternal }}$, the distance between $n_{\text {johny,olson }}$ and $n_{\text {keep }}$ must be greater than the distance between $n_{\text {johny,olson }}$ and $n_{\text {eternal }}$ violating the JKT conditions. Thus, $n_{\text {johny,olson }}$ can only be appended as a child of $n_{\text {keep }}$. Finally, $n_{\text {love }}$ is inserted in the same vertex as $n_{\text {eternal }}$, yielding the $J K T$ of Figure 5.

\subsection{Selection of top- $K$ databases}

We describe the selection of the top- $K$ databases, considering both AND and OR semantics. AND semantics require each result to contain all query keywords. According to OR semantics a result may include only a subset of the keywords. Let $\kappa$ be the term cardinality of a query $q$, and $|c g|$ be the largest number of query keywords that yield a candidate graph in the $K R G$ of a database $D B$. If $|c g|<\kappa$, then according to Theorem 5.1, $D B$ cannot produce any result for $q$ based on AND semantics, and can be safely eliminated. On the other hand, for OR semantics, $D B$ may still constitute a candidate for search. For two databases $D B_{1}$ and $D B_{2}$, if $\left|c g_{1}\right|>\left|c g_{2}\right|$, then $D B_{1}$ is, in general, expected to be a better candidate than $D B_{2}$. However, in certain cases $D B_{2}$ may rank higher, if the query keywords have large weights in its $K R G$, and occur in tuples close to each other.

The basic functionality of database selection is shown in Algorithm 2, hereafter referred to as SelectDB. SelectDB uses a set $S_{|c g|}$ to store the ids of all databases that contain candidate graphs with $|c g|$ keywords $(|c g| \leq \kappa)$. The 


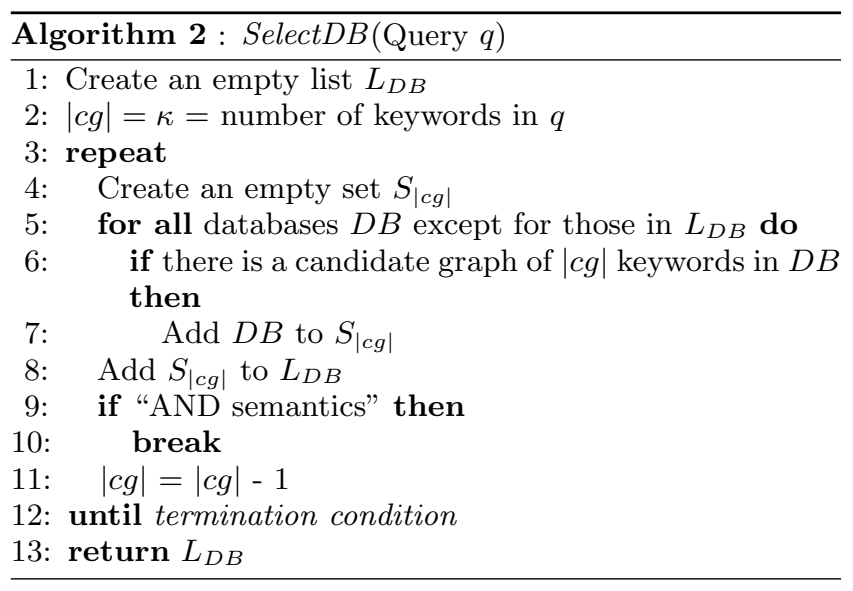

individual sets for various values of $|c g|$ are maintained in a set list $L_{D B}$. The algorithm first finds databases containing candidate graphs with all query keywords. The ids of these databases are inserted into $S_{\kappa}$ and $L_{D B}$. If the query is based on AND semantics, SelectDB terminates and returns $L_{D B}$ to a scoring mechanism (to be discussed shortly).

In case of OR semantics, the termination condition is flexible. At one extreme, a user may want to rank all databases containing one or more keywords. At the other extreme, another user may wish to rank only the $K$ databases that contain the maximum number of keywords. To accommodate this flexibility, line 12 does not specify an explicit termination condition ${ }^{6}$. Assume that after finding databases with $|c g|=\kappa$, we want to retrieve databases with $|c g|=\kappa-1$. Select $D B$ derives $\kappa$ new queries of length $\kappa$-1, by removing each keyword in turn from $q$ (for simplicity, the details of this process are omitted from the pseudo-code). All databases, except for those already in $L_{D B}$, are evaluated against the new queries, and those containing candidate graphs are inserted into $S_{\kappa-1}$ and $L_{D B}$. This process is repeated for $\kappa-2$ and so on, until the termination condition. The list $L_{D B}$ contains the candidate databases in decreasing order of their $|c g|$, for facilitating ranking based on the size of the candidate graphs.

Given the wealth of information maintained by the $K R G$, $G-K S$ can integrate a wide variety of scoring mechanisms. Adopting an IR-based methodology, we calculate the similarity between a database and a query as:

$$
\operatorname{Score}(D B, q)=\sum_{\left\{k_{i}, k_{j}\right\} \subseteq q, i \neq j} \operatorname{Score}\left(k_{i}, k_{j}\right)
$$

where $\left\{k_{i}, k_{j}\right\}$ is a pair of terms in $q$, and $\operatorname{Score}\left(k_{i}, k_{j}\right)$ is their accumulated score calculated as:

$$
\operatorname{Score}\left(k_{i}, k_{j}\right)=w_{i} \cdot w_{j} \cdot \sum_{d} w_{i j}(d)
$$

Recall from Section 3, that $w_{i}$ and $w_{j}$ are the weights of nodes $n_{i}$ and $n_{j}$, representing terms $k_{i}$ and $k_{j}$, respectively, while $w_{i j}(d)$ is the weight of the edge connecting $n_{i}$ and $n_{j}$ at distance $d$. $G$-KS computes the score of each database in $L_{D B}$ based on the above equations and returns the top$K$ with the highest scores. Note that, for AND semantics, $L_{D B}$ may contain contain fewer than $K$ databases. In such

${ }^{6}$ Details about the concrete implementation are discussed in Section 6. cases, the benefits of $G-K S$ are even more important as it saves redundant search in numerous databases that cannot contain any results.

\subsection{Comparison of $G-K S$ and $M-K S$}

In this section, we discuss the differences between $G-K S$ and $M-K S$, assuming, for consistency, that $M-K S$ uses the SUM function ${ }^{7}$ to calculate the similarity score between a database and a query. Specifically, $\operatorname{Score}(D B, q)$ is also given by Equation 1, but the score of a keyword pair is:

$$
\operatorname{Score}\left(k_{i}, k_{j}\right)=\sum_{d} \frac{p f_{S U M i j}(d)}{d+1}
$$

where $p f_{S U M i j}(d)$ is the total number of occurrences of the pair $\left(k_{i}, k_{j}\right)$ at distance $d$ calculated as:

$$
p f_{S U M i j}(d)=\sum_{t_{x}, t_{y}} p f_{i j}\left(t_{x}, t_{y}, d\right)
$$

$M-K S$ considers all keyword terms to be equally important, and simply counts the number of term co-occurrences to compute the weights of distance relationships in the matrix. Therefore, it cannot deal with the problem of popular terms, mentioned in Section 2.1. For instance, a database that contains frequent co-occurrences for a pair of query terms would achieve a large score, although these co-occurrences do not have high discriminating value (indeed, because of their frequency). In contrast, $G-K S$ normalizes the weights of nodes and edges, solving the problem of popular terms.

The second difference between the two methods is due to the use of compound nodes in $K R G$. Compound nodes represent multiple terms that occur a single time in the database, and at the same tuple. This concept leads to effective compression of the $K R G$, since all terms in a compound node share the same set of distance relationships. In contrast, $M$ $K S$ explicitly represents the distance relationship between all pairs of terms, without utilizing any compression mechanism. The resulting $K R M$ has more entries than the corresponding $K R G$, consumes more space and incurs higher cost during query processing.

The third, and most important, difference regards the effectiveness of pruning. $M-K S$ simply uses the binary relationships of queried keywords in $K R M$ to calculate similarity scores. Consequently, it incurs a large number of false positives for queries with more than two keywords. In contrast, the concept of the candidate graph allows $G-K S$ to rise above binary relationships, and treat the query terms holistically. As we show in the next Section, this leads to significant gains in terms of precision and recall.

\section{EXPERIMENTAL EVALUATION}

We assume a client-server architecture where the server stores summaries of several databases. A client issues a query $q$ to the server, which returns the part of the summary that is relevant to $q$, i.e., the weights of the query terms and their distance relationships. Given this informa-

\footnotetext{
${ }^{7}[26]$ suggests using the SUM, PROD, MAX or MIN functions for combining the pairwise scores. PROD and MAX have a similar effect to SUM, while MIN cannot capture OR semantics.
} 


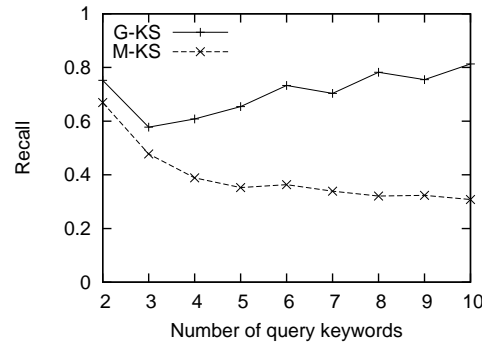

(a) Recall

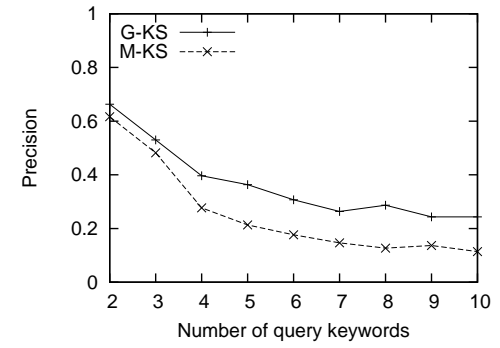

(b) Precision

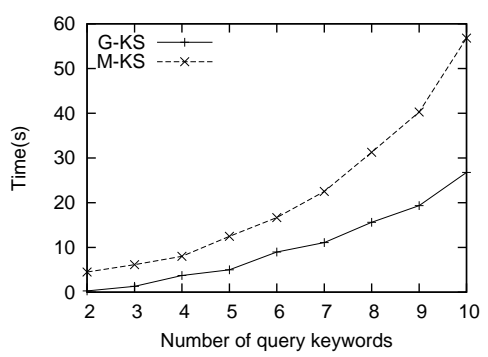

(c) Query response time

Figure 7: Recall, precision, and query response time versus $\kappa$

tion, the client selects the top- $K$ databases locally ${ }^{8}$. In addition to responding to queries, the server has to construct and maintain the summaries in the presence of database updates. The server uses MySQL 4.1.7 to store and retrieve the summaries. Specifically, each KRG is represented by: (1) a node table where a tuple $\left(n_{i}, w_{i}\right)$ signifies that the weight of node $n_{i}$ is $w_{i},(2)$ a term table where a tuple $\left(k_{j}, n_{i}\right)$ represents that term $k_{j}$ is mapped to node $n_{i}$, and (3) an edge table, where a tuple $\left(n_{i}, n_{j}, d, w\right)$ indicates that nodes $n_{i}$ and $n_{j}$ are connected at distance $d ; w$ is the weight of the distance relationship. The client is an Intel Pentium $4 \mathrm{CPU}$ $2.4 \mathrm{GHz}$ and the server is an Intel Xeon MP CPU 3.00 GHz. The simulator is implemented using Java JDK 5.0.

Due to the unavailability of multiple real databases, we follow a methodology similar to [26]. Specifically, we decompose DBLP [6] into distinct databases according to bibliography types such as articles, proceedings, books. Since some of the resulting databases (e.g., articles, proceedings) are rather large, we decompose them further, to derive a total of 81 databases, each containing in the order of 50,000 records residing in 4-5 tables. We evaluate $G-K S$ against $M-K S[26]$ as there is no other competitor in the literature. The client-server framework remains the same, except that the server maintains KRMs instead of KRGs. G-KS applies the compression technique of Section 3.3 to reduce the $K R G$ size. Section 6.1 compares the two methods on the preprocessing overhead. Section 6.2 focuses on the effectiveness and efficiency of query processing. Section 6.3 investigates the effect of delayed updates on the data summaries.

\subsection{Pre-processing overhead}

Table 2 illustrates the average construction time and space consumption per summary, assuming that the maximum distance relationship $\delta$ preserved in each summary is 4 . $G$-KS is faster (31.54\%) and requires less disk space (17.24\%) than $M-K S$ because of the compression of KRGs. For instance, given a database of 22,423 distinct terms and 53,214 tuples, $G$ - $K S$ creates a total of $20,308,927$ distance relationships in the compressed $K R G$, whereas $M-K S$ creates a total of $24,661,881$ relationships in the $K R M$.

\subsection{Query processing}

Since the probability for two random keywords to have an interesting association is very low, we generate queries con-

${ }^{8}$ We chose this architecture because of its flexibility, e.g., each client may implement the scoring mechanisms of its choice. Alternatively, query processing can take place at the server.
Table 2: Pre-processing cost

\begin{tabular}{|l|l|l|}
\hline & Time (Hours) & Space (MB) \\
\hline$M-K S$ & 7.26 & 438.12 \\
\hline$G-K S$ & 4.97 & 362.59 \\
\hline Improvement & $31.54 \%$ & $17.24 \%$ \\
\hline
\end{tabular}

taining terms that are pair-wisely related in at least some KRGs. Furthermore, we assume OR semantics, so that a result may miss some query keywords. $G-K S$ uses the techniques of Section 5.3 to rank the databases. The termination condition of Algorithm 2 is: $\left|L_{D B}\right| \geq K$, i.e., Select $D B$ stops at a value of $|c g|$ such that the number of databases with candidate graphs is at least $K$. We also apply SelectDB for $M-K S$ since the original version of the method cannot handle OR semantics. $M-K S$ implements the scoring functions of Section 5.4.

The experiments focus on the efficiency and the effectiveness of the methods. Efficiency refers to the query response time, which is the duration from the instance that the client sends a query $q$ to the server, until the time it produces the top- $K$ databases for $q$. This includes (1) the retrieval of summaries at the server, and the extraction of the relevant information for $q,(2)$ the network latency for transmitting this information to the client, and (3) the processing cost at the client. The network latency is very small and similar in both $G-K S$ and $M-K S$; thus, the response time and the performance difference between the techniques is dominated by the other two factors.

Effectiveness refers to the concepts of precision and recall. In order to measure effectiveness, we use a brute-force (BF) method that, given $q$, receives the top-50 results from every database using the method of [16]. Let $M$ be the maximum number of keywords in any result $(M \leq \kappa)$. BF selects the top- $K$ databases based on the cardinality of the results containing $M$ keywords. For instance, if $D B_{1}$ returns 10 results with $M=5$ keywords, and $D B_{2}$ returns 20 results with 4 keywords, then $D B_{1}$ obtains a higher score than $D B_{2}$. Let $K_{B F}(M), K_{G K S}(M)$ be the total number of results with $M$ keywords in $\mathrm{BF}$ and $G-K S$, respectively. Then, recall is defined as $K_{G K S}(M) / K_{B F}(M)$. Precision is the ratio of the number of selected databases having results with $M$ keywords in $G$ - $K S$ over $K$. The recall and precision of $M-K S$ are defined in the same way.

The experiments involve three parameters: the query length $\kappa$, the maximum distance $\delta$ between any two terms in the summary, and the number $K$ of selected databases. Table 3 summarizes the default and range of values of these param- 


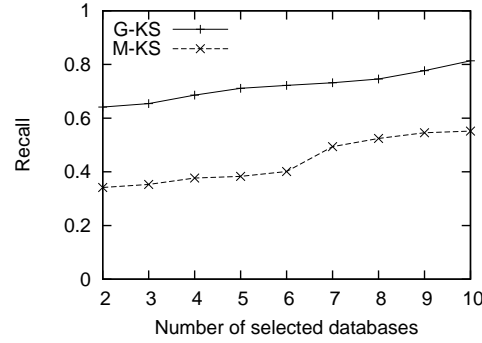

(a) Recall

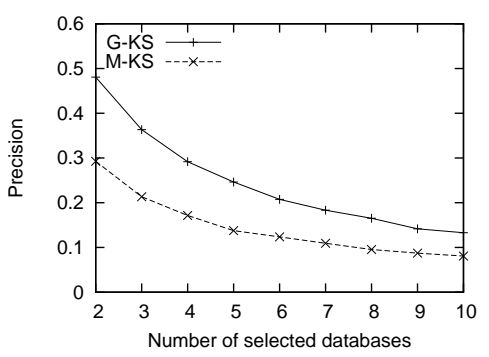

(b) Precision

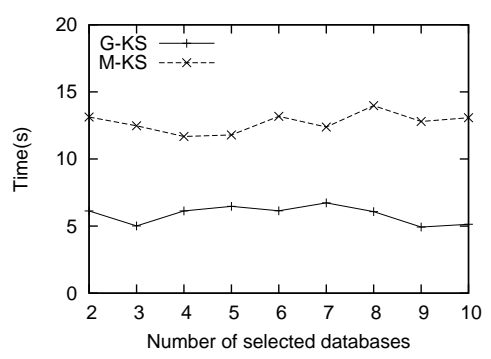

(c) Query response time

Figure 8: Recall, precision and query response time versus $K$

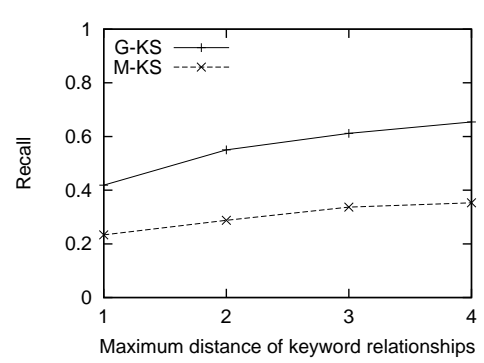

(a) Recall

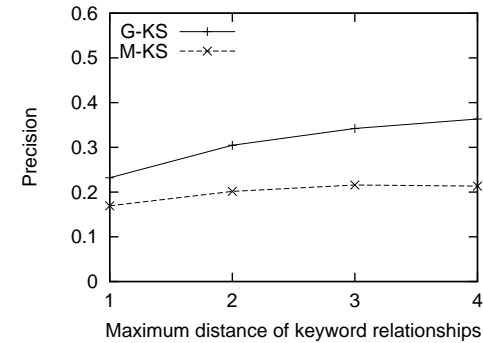

(b) Precision

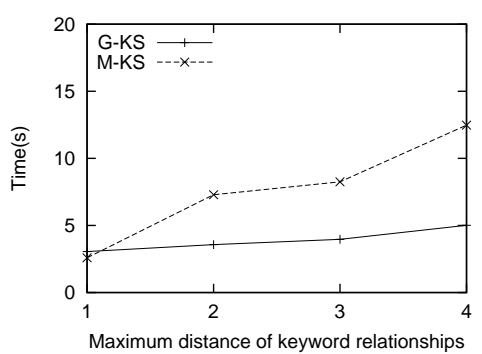

(c) Query response time

Figure 9: Recall, precision and query response time versus $\delta$

Table 3: Query parameters

\begin{tabular}{|l|c|c|}
\hline Parameter & Default value & Range of values \\
\hline$\delta$ & 4 & $1-4$ \\
\hline$\kappa$ & 5 & $2-10$ \\
\hline$K$ & 3 & $2-10$ \\
\hline
\end{tabular}

eters. For each experiment we vary one parameter, while setting the other two to their default values. A number of 100 queries are executed and the displayed results are the average results from executing these queries.

Figure 7 evaluates recall, precision and response time as a function of $\kappa$. When the number of query keywords increases, the recall of $M-K S$ drops due to false positives incurred by the binary filtering mechanism. On the other hand, $G-K S$ checks relationships holistically through Validate $S G$ and its recall grows with $\kappa$. Note that for large values of $\kappa$, there may be less than $K$ databases with the maximum number of keywords, leading to relatively low precision. However, the high value of recall implies that $G-K S$ obtains most of these databases. In addition to effectiveness, $G-K S$ outperforms $M-K S$ in terms of the efficiency. This result may initially appear counter-intuitive since $G-K S$ performs the additional step of filtering through candidate graphs. However, the total processing cost is dominated by the retrieval of keyword relationships in the summaries. Since each $K R G$ is significantly smaller that the corresponding $K R M$, the retrieval cost of $G-K S$ is lower.

Figure 8 measures the effect of the number $K$ of selected databases. As expected, the recall increases with $K$ since more databases are likely to produce more results. On the other hand, the precision drops since a larger percentage of these results is irrelevant. Again $G-K S$ has an important advantage over $M-K S$ on both measures. The total processing cost is insensitive to the value of $K$, because the server transmits the same amount of information to the client, independently of the number of databases to be selected. Therefore, the overhead of summary retrieval, which is the dominant factor on the response time, does not change.

Figure 9 studies the effect of $\delta$. A large $\delta$ implies detailed information in the summary. $G$ - $K S$ utilizes the additional information to improve both precision and recall. On the other hand, the same is not necessarily true for $M$ $K S$, as more relationships at higher distances may increase the number of false positives, misleading the system to overestimate the usefulness of a database. The efficiency drops because the summary size is proportional to $\delta$ and larger summaries lead to longer retrieval times for keywords relationships.

\subsection{Effect of delayed updates}

As discussed in Section 4.2, to avoid the cost of frequent revisions, the $K R G$ of a database may be updated in batches, instead of after every record insertion and deletion. This experiment evaluates the effectiveness as a function of the number of updates that have not been reflected in the summary. An update deletes a random record from one database, and inserts it in another one. Figure 10 shows precision and recall versus the percentage of the database size that has been modified. As expected, the effectiveness of both $M-K S$ and $G-K S$ decreases with the percentage of the modified data. Note that the results of $G-K S$ when $10 \%$ of the data have been modified, are better than those of $M-K S$ before any updates.

\section{CONCLUSION}

This paper proposes $G$ - $K S$, a method that selects the 


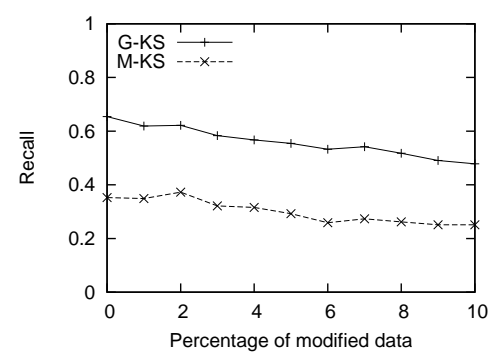

(a) Recall

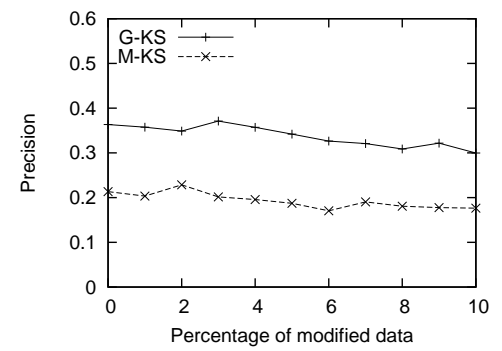

(b) Precision

Figure 10: Recall and precision versus data modification

top- $K$ databases for processing a relational keyword search query. $G-K S$ summarizes each database as a keyword relationship graph, where nodes correspond to terms, and edges capture distance relationships. Both nodes and edges are weighted according to state-of-the-art IR techniques in order to support a variety of scoring functions. Based on the $K R G, G-K S$ applies an intricate algorithm to identify and eliminate non-promising databases. As opposed to the previous work that is based only on binary relationship between terms, $G$ - $K S$ considers all query keywords as a whole in order to minimize the number of false positives. An extensive experimental evaluation confirms the superiority of $G-K S$ in terms of effectiveness, efficiency, processing and pre-processing overhead.

\section{REFERENCES}

[1] S. Agrawal, S. Chaudhuri, and G. Das. DBXplorer: A system for keyword-based search over relational databases. In Proceedings of ICDE, 2002.

[2] G. Bhalotia, A. Hulgeri, C. Nakhe, S. Chakrabarti, and S. Sudarshan. Keyword searching and browsing in databases using banks. In Proceedings of ICDE, 2002.

[3] J. P. Callan, Z. Lu, and W. B. Croft. Searching distributed collections with inference networks. In Proceedings of SIGIR, 1995.

[4] G. Cao, J.-Y. Nie, and J. Bai. Integrating word relationships into language models. In Proceedings of SIGIR, 2005.

[5] S. Cohen. XSEarch: A semantic search engine for XML. In Proceedings of $V L D B, 2003$.

[6] DBLP. http://dblp.uni-trier.de.

[7] C. Fellbaum, editor. Wordnet: An Electronic Lexical Database. MIT Press, 1998.

[8] J. Gao, J.-Y. Nie, G. Wu, , and G. Cao. Dependence language model for information retrieval. In Proceedings of SIGIR, 2004.
[9] L. Gravano, H. Garcia-Molina, and A. Tomasic. GlOSS: Text-source discovery over the internet. $A C M$ Transactions on Database Systems (TODS), 24(2):229-264, 1999.

[10] L. Guo, F. Shao, C. Botev, and J. Shanmugasundaram. XRANK: Ranked keyword search over XML documents. In Proceedings of SIGMOD, 2003.

[11] H. He, H. Wang, J. Yang, and P. S. Yu. BLINKS: Ranked keyword searched on graphs. In Proceedings of SIGMOD, 2007.

[12] V. Hristidis, L. Gravano, and Y. Papakonstantinou. Efficient IR-style keyword search over relational databases. In Proceedings of VLDB, 2003.

[13] V. Hristidis and Y. Papakonstantinou. DISCOVER: Keyword search in relational databases. In Proceedings of $V L D B, 2002$.

[14] V. Kacholia, S. Pandit, S. Chakrabarti, S. Sudarshan, R. Desai, and H. Karambelkar. Bidirectional expansion for keyword search on graph databases. In Proceedings of $V L D B, 2005$.

[15] Y. Li, C. Yu, and H. V. Jagadish. Schema-Free XQuery. In Proceedings of $V L D B, 2004$.

[16] F. Liu, C. Yu, W. Meng, and A. Chowdhury. Effective keyword search in relational databases. In Proceedings of SIGMOD, 2006.

[17] Z. Liu and Y. Chen. Identifying meaningful return information for XML keyword search. In Proceedings of SIGMOD, 2007.

[18] Y. Luo, X. Lin, W. Wang, and X. Zhou. SPARK: Top-k keyword query in relational databases. In Proceedings of SIGMOD, 2007.

[19] A. Markowetz, Y. Yang, and D. Papadias. Keyword search on relational data streams. In Proceedings of SIGMOD, 2007.

[20] R. Nallapati and J. Allan. Capturing term dependencies using a language model based on sentence trees. In Proceedings of CIKM, 2002.

[21] S3: Scalable, Shareable and Secure P2P Based Data Management System. http://www.comp.nus.edu.sg/ ^s3p2p.

[22] G. Salton, A. Wong, and C. S. Yang. A vector space model for automatic indexing. Communications of the $A C M, 18(11): 613-620,1975$.

[23] M. Sayyadian, H. LeKhac, A. Doan, and L. Gravano. Efficient keyword search across heterogeneous relational databases. In Proceedings of ICDE, 2007.

[24] S. K. M. Wong, W. Ziarko, V. V. Raghavan, and P. C. N. Wong. On modeling of information retrieval concepts in vector spaces. ACM Transactions on Database Systems (TODS), 12(3):299-321, 1987.

[25] Y. Xu and Y. Papakonstantinou. Efficient keyword search for smallest LCAs in XML databases. In Proceedings of SIGMOD, 2005.

[26] B. Yu, G. Li, K. Sollins, and A. K. H. Tung. Effective keyword-based selection of relational databases. In Proceedings of SIGMOD, 2007.

[27] B. Yuwono and D. L. Lee. Server ranking for distributed text retrieval systems on the internet. In Proceedings of DASFAA, 1997. 